\title{
Kernkompetenz Kundenkontakt nutzen
}

\author{
Michaela Weiß und Peter Weiß
}

\begin{abstract}
Etablierte soziale Organisationen können beim Persönlichen Budget mit einem Pfund wuchern, das neue Anbieter nicht haben: mit dem direkten Kontakt zum Nutzer und dem Wissen um dessen individuelle Situation. Wie dieser Vorteil organisatorisch umgesetzt werden kann, zeigt das Beispiel eines Leistungsanbieters aus Süddeutschland.
\end{abstract}

»Sozialteam« ist eine gemeinnützige Gesellschaft mit beschränkter Haftung und Mitglied im Paritätischen Wohlfahrtsverband. Dezentral erbringt die Non-Profit-Organisation an sieben Standorten in Bayern und Sachsen, in sogenannten Soziotherapeutischen Zentren abgestufte Dienste und Betreuungsleistungen für psychisch kranke und suchtkranke Menschen in den Bereichen niedrigschwellige Betreuung und Beratung, Wohnen, Beschäftigung und Zuverdienst (Internet http://www.sozialteam.de). Wir verstehen uns als Dienstleistungsunternehmen unserer Nutzer und Nutzerinnen. Entsprechend seiner Kernkompetenz hat sich Sozialteam auf Budgetberatung und Budgetangebote für die Zielgruppen psychisch kranke und suchtkranke Menschen spezialisiert.

\section{Vorbereitung}

Schon vor einigen Jahren begannen bei Sozialteam im Rahmen der Strategieplanungen die Vorbereitungen auf das Persönliche Budget. Dabei spielte neben einer intensiven Auswertung der Ergebnisse und Erfahrungen aus den Modellprojekten eine gemeinsame strategische Planung zu diesem neuen Finanzierungsmodell mit der mittleren Leistungsebene eine große Rolle. Zielsetzung war dabei von Anfang an sowohl Budgetberatung anzubieten, als auch potenziellen Kunden Angebote im Rahmen des Persönlichen Budgets zu unterbreiten. Die konkreten Vorbereitungen liefen dabei sowohl auf der Ebene der Organisation als auch der potenziellen Budgetberaterinnen und Budgetberatern.

Mit einer intensiven Organisationsentwicklung wurden insbesondere auf fünf aktuelle Herausforderungen eingegangen:

\footnotetext{
1 Herausforderung: Das Instrument »Fachleistungs1. stunden « musste erprobt werden. In Zusammenarbeit mit einem Leistungsträger führt Sozialteam ein Modellprojekt zur Einführung und Umsetzung von
}

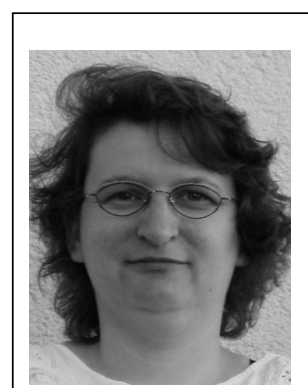
Michaela Weiß (36) hat Sozial- pädagogik an der Fachhochschule Regensburg studiert. Berufs- begleitend hat sie sich an der Deutschen Fundraisinig-Akade- mie zur Fundraising-Managerin (FA) weitergebildet. Sie ist seit über zehn Jahren bei Sozialteam tätig, aktuell als Projektleiterin.

Hier bildet ein Schwerpunkt ihrer Tätigkeit die Fachberatung mehrerer Einrichtungen des gemeinnützigen Trägers im Bereich der Sozialpsychiatrie. Daneben verantwortet sie bei Sozialteam $u$. a. die Bereiche Marketing und Fundraising.

E-Mail Michaela.Weiss@sozialteam.de

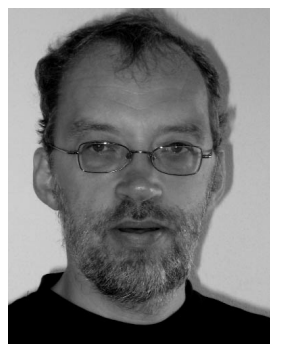

Peter Weiß (47) ist Sozialpädagoge und Sozialwirt. Berufsbegleitend hat er sich zum Qualitätsmanager und NPO-Controller fortgebildet. Er hat vor über 16 Jahren Sozialteam mitbegründet. Er leitet als Geschäftsführer den Fachbereich Soziotherapeutische Einrichtungen bei Sozialteam. Darunter sind die Angebote für psychisch kranke und suchtkranke Menschen gebündelt. In der dieser Funktion ist er $u$. a. für Fragen der strategischen Planung und des Controllings zuständig. E-Mail Peter.Weiss@sozialteam.de

Fachleistungsstunden bei psychisch kranken Menschen im ambulant betreuten Wohnen durch. Mit diesem Projekt konnten wertvolle Informationen zu folgenden Bereichen gewonnen werden, die auch bei Angeboten zum Persönlichen Budget so oder ähnlich eine wichtige Rolle spielen:

- Fragen der internen Arbeitsorganisation (Beispiel: »Welche indirekten Arbeitsstundenanteile sind nötig, um eine Face-to-Face-Leistung erbringen zu können?«)

- Wie lässt sich der Personaleinsatz so flexibel organisieren, dass zum einem eine dauerhafte (z. B. Vertretungsfragen), aber auch eine sehr nutzerorientierte Betreuung 
gewährleistet werden kann (flexible, zeitnahe Vertretungen, selbstverantwortete Einsatzplanung von Mitarbeiterinnen und Mitarbeitern etc.)?

- Wie lassen sich diese fachlichen Ansprüche mit der Vielzahl arbeitsrechtlicher und betriebsverfassungsrechtlicher Regelungen vereinbaren?

- Fragen der flexiblen einrichtungsübergreifenden Erbringung von Leistungen. Da die Zielgruppe des Modellprojektes psychisch kranke Menschen waren, die aufgrund ihres Betreuungsumfanges normalerweise die Betreuung in einer Wohnstätte benötigen, war es nötig, jeweils individuelle Leistungspakte zu schnüren, in denen auch Leistungen aus verschiedenen Einrichtungen mit abgerufen werden.

2 Herausforderung: Flexible Personalpools müssen • eingerichtet werden. Schon im Vorfeld wurde langfristig in allen Einrichtungen ein flexibler Personalpool durch eine Vielzahl von aufeinander abgestimmten Maßnahmen geschaffen (Einbezug von Honorarkräften, Arbeitszeit auf Abruf u. a. m), der sowohl einen zeitlich als auch inhaltlich flexiblen Einsatz von Mitarbeiterinnen und Mitarbeitern ermöglicht.

3. Herausforderung: Das Innovationsmanagement in 3. der Organisation musste gestärkt werden. Bei Sozialteam wird von Anfang an viel Wert auf die Stärkung der eigenen Innovationskraft gelegt. Dies findet seinen Ausdruck beispielsweise in jährlichen Strategieklausuren mit der obersten Führungsebene und dem Einbezug der Mitarbeitenden in den Einrichtungen in konzeptionelle Weiterentwicklungsprozesse. Im Vorfeld der gesetzlichen Veränderungen beim Persönlichen Budget am 1. Januar 2008 wurden von der Geschäftsführung alle Mitarbeiterinnen und Mitarbeiter über diese neue Leistungsform informiert. Zielsetzung war dabei, die Chancen, die sich durch das Persönliche Budget ergeben, aufzuzeigen, in der Branche bestehende Ängste abzubauen und den Mitarbeiterinnen und Mitarbeitern die Sicherheit zu vermitteln, dass ihre Geschäftsführung gut vorbereitet in dieses neue Angebotsfeld geht.

4. Herausforderung: Das Risikomanagement musste ausgebaut und weiter qualifiziert werden. Da durch das Persönliche Budget und die damit einhergehenden größeren Erlösschwankungen - die mittelfristig weniger voraussehbar als bei der Sachleistung sind - das betriebswirtschaftliche Risiko von Anbietern im Rahmen des Persönlichen Budgets sicher steigen wird, wurde gezielt in den letzten Jahren das Risikomanagement ausgebaut, um so schneller auf solche zu erwartende Schwankungen reagieren zu können.

5 Herausforderung: Budgetberaterinnen und Budget5 . berater mussten ausgewählt werden. Anhand eines Qualifikationsprofils wurde entschieden, welche Mitarbeitenden die Funktion von Budgetberatern an den jeweiligen Sozialteam-Standorten übernehmen sollten. Dabei waren wichtige Qualifikationen: Erfahrungen in der Beratung von Nutzerinnen und Nutzern sowie deren gesetzlich bestellten Betreuerinnen und Betreuer, Verhandlungserfahrungen mit Leistungsträgern, guter Einblick und persönliche Vernetzung im regionalen psychosozialen Netz, nutzerorientiertes Dienstleistungsverständnis.
Die ausgewählten Budgetberaterinnen und Budgetberater wurden intern und durch den Paritätischen Wohlfahrtsverband intensiv fortgebildet zu den Themenkomplexen rechtliche Rahmenbedingungen des Persönlichen Budgets, Beratungs- und Verhandlungskompetenz und Beschreibung von Angeboten im Rahmen des Persönlichen Budgets.

\section{Umsetzung}

Die Realisierung der Planungen wurde in folgenden Schritten angegangen:

- Von den Einrichtungsleitungen wurden mögliche Angebote im Rahmen des Persönlichen Budgets beschrieben. Dabei wurde jeweils Bezug genommen auf die vorhandenen Kernkompetenzen, die in diesen Einrichtungen und Diensten vorhanden sind. Dies beinhaltet auch einzelne Leistungen, die bis jetzt als Teile von Komplexleistungen erbracht wurden.

- Durch die Geschäftsführung wurden einige Angebote modellhaft verpreislicht. Vereinfacht dargestellt bildet die Basis der Verpreislichung die jeweils identifizierten Einzelkosten der Leistung (Personal- und Sachkosten) sowie ein Aufschlag für die Gemeinkosten (z. B. Marketing, Verwaltung).

- Eine weitere Aufgabe stellte die finanzielle Absicherung der Budgetberatung dar. Da diese bis jetzt in der Regel nicht finanziert wird, war es Aufgabe, dafür Finanzmittel bereitzustellen. Dies erfolgte vor allem durch die Akquise von Spendenmitteln, Bußgeldern etc.

- In einem Marketingplan wurde die zeitliche Abfolge des Bekanntmachens der Budgetberatung auf der einen Seite sowie der Bereitstellung von Angeboten im Rahmen des Persönlichen Budgets auf der anderen Seite systematisch geplant.

\section{Erfahrungen}

Nach den ersten Monaten Budgetberatung kann bereits über manch überraschende Erfahrung berichtet werden. Besonders auffällig ist, dass Aufklärungs- und Informationsarbeit über gesetzliche Grundlagen des Persönlichen Budgets noch immer sehr im Vordergrund stehen. Unsere Erfahrung ist, dass das Persönliche Budget als Wahlmöglichkeit bei den betroffenen behinderten Menschen trotz Kampagnen seitens der Politik - noch nicht oder nur sehr verschwommen bei den Menschen mit Behinderung angekommen ist. Eine wichtige Rolle spielen unserer Erfahrung nach Fragen der Heranziehung von Einkommen und Vermögen sowie Nachweispflichten beim Persönlichen Budget.

Da wir schwerpunktmäßig psychisch kranke und suchtkranke Menschen beraten, die oft einen gesetzlich bestellten Betreuer zur Seite bestellt bekommen haben, spielt bei Sozialteam auch die Beratung dieser Berufsgruppe eine wichtige Rolle. Hier stellen wir viele verständliche Vorbehalte fest, da durch die Hilfe bei der Beantragung und 
Verwaltung des Persönlichen Budgets ihrer Betreuten ein unbezahlter Mehraufwand befürchtet wird. Leider ist diese Befürchtung nur zu berechtigt, hier muss sich etwas in der Honorierung der Betreuertätigkeiten ändern, wenn das Persönliche Budget auch bei behinderten Menschen, die unter gesetzlicher Betreuung stehen, größere Relevanz finden soll - oder es muss alternativ eine finanzierte Budgetassistenz für den Budgetempfänger eingeführt werden.

Bei den Leistungsträgern ist auch nach fast einem Jahr der rechtsverbindlicher Einführung des Persönlichen Budgets ein unterschiedlicher Entwicklungsstand zu diagnostizieren. Die Bandbreite reicht dabei von Unwissenheit, dem Versuch, solche Geldleistungen eher abzuwehren bis zur Bereitschaft, individuelle Lösungen für betroffene Menschen zu finden. Ein großes Manko stellt die Tatsa-

gen »Hilfeverständnis « hinterfragen lassen und ihr oft noch unzureichendes Dienstleistungsverständnis entwickeln. Dieses muss fachliche Standards auf der Basis und unter ständigen Einbezug der individuellen Wünsche der Budgetnehmerinnen und Budgetnehmer umsetzen. Das Persönliche Budget bietet zudem Organisationen die Möglichkeit, neue Angebote zu erfinden und bestimmten Zielgruppen (wie beispielsweise für Personen, die diskriminierend als »Systemsprenger « definiert werden) bedarfsorientierte fachlich adäquate Lösungen anbieten zu können.

- Risiken und Grenzen: Bei einigen wenigen Leistungsträgern ist durchaus die Tendenz zu sehen, mittels des Persönlichen Budgets, Qualität und Preise bisheriger Versorgungsangebote zu drücken oder Leistungen, die

bis jetzt unbestritten im

\section{»Durch das Persönliche Budget wird es bei vielen Anbietern zu größeren Erlösschwankungen kommen«}

Rahmen von Komplexleistungen von Fachkräften erbracht wurden, nun im Rahmen der Einzelleistung

che dar, dass viele Leistungsträger der Eingliederungshilfe noch keine oder nur sehr unzureichende Erfahrungen mit Fachleistungsstunden und flexiblen Betreuungsschlüsseln haben. Gut bewährt hat sich die Kooperation mit den Leistungsträgern, die schon über umfangreiche Erfahrungen mit Hilfeplankonferenzen verfügen, an denen seitens des Leistungsträgers auch die Fachdienste entscheidungsbefugt teilnehmen.

Für das Marketing von überragender Bedeutung ist die Erfahrung, dass in Flyern vorgestellte Angebote nur als Anregung dienen können. Wichtig ist es, in Gesprächen mit den potenziellen Budgetnehmern individuelle Angebote zu stricken, die die Grenzen vollstationär und ambulant, aber auch Leistungsanbietergrenzen überschreiten. Basis dafür muss dort, wo es sinnvoll ist, auch die Einbeziehung des sozialen Netzwerkes sein.

\section{Resümee}

Wie viele Neuerungen birgt das Persönliche Budget für Leistungserbringer sowohl Chancen wie Gefahren.

- Chancen: Durch das persönliche Budget ist eine Autonomiestärkung der betroffenen Leistungsempfänger festzustellen. Mit Hilfe eines oft vereinfachten Antragsverfahren (im Vergleich zum Antrag auf Sachleistungen) und der Teilnahme an Budgetkonferenzen können sie ihre individuellen Wünsche zur Deckung ihres persönlichen Hilfebedarfs direkt dem Leistungsträger gegenüber vortragen. Aber auch durch die Tatsache, nun selber den Leistungserbringer wählen und bezahlen zu können, steigt nicht nur das Selbstbewusstsein behinderter Menschen, sondern auch ihr Status als ernstzunehmender Kunde. Leistungserbringer sind gezwungen - und haben aber auch die fachliche Chance -, individuellere, zielgerichtere Angebote zu entwerfen und umzusetzen. Professionelle müssen sich in ihrem bisheri- von Hilfskräften erbringen zu lassen. Für die Leistungsanbieter werden Schwankungen in der Nachfrage ihres Angebotes zunehmen. Durch das Persönliche Budget entsteht jetzt schon, rein quantitativ, eine größere Anzahl von Konkurrenten. Viele kleine neue Anbieter drängen auf dem Markt, die oft beweglicher sind als traditionelle große Anbieter. Daneben ist in Zukunft von bedeutend höheren Schwankungen in Auslastung und Erlösen auszugehen.

Damit das Persönliche Budget mehr an Bedeutung gewinnen kann, sind einige Schritte nötig:

- Die Budgetassistenz muss ausreichend honoriert werden.

- Die Verwaltung des Persönlichen Budgets durch gesetzlich bestelle Betreuer muss, beispielsweise durch höhere Aufwandspauschalen, angemessen vergütet werden. Nur so kann gesichert werden, dass schwächere behinderte Menschen ausreichend an den Möglichkeiten des Persönlichen Budgets partizipieren können.

- Sinnvoll wären - im Sinne des Verbraucherschutzes und der Fachlichkeit des Angebotes - einheitliche Standards für Leistungserbringer zu entwerfen, die insbesondere die Fragen der Qualifikation der Leistungserbringer, der Sicherstellung der Dauerhaftigkeit des Angebotes über den gesamten bewilligten Zeitraum u. a. m. sichern. 